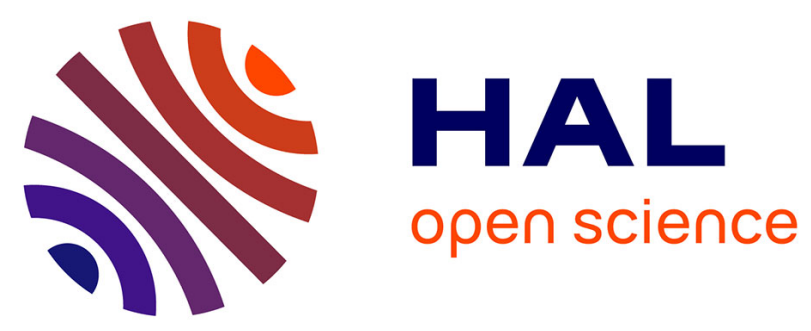

\title{
Métastase mammaire d'un carcinome médullaire thyroïdien : un challenge diagnostique
}

Charlotte Gardair, Philippe Drabent, Patrick Charveriat, Philippe Bertheau, Laurence Cahen-Doidy, Anne de Roquancourt

\section{To cite this version:}

Charlotte Gardair, Philippe Drabent, Patrick Charveriat, Philippe Bertheau, Laurence Cahen-Doidy, et al.. Métastase mammaire d'un carcinome médullaire thyroïdien: un challenge diagnostique. Annales de Pathologie, 2019, 39, pp.9 - 13. 10.1016/j.annpat.2018.07.004 hal-03486693

\section{HAL Id: hal-03486693 https://hal.science/hal-03486693}

Submitted on 20 Dec 2021

HAL is a multi-disciplinary open access archive for the deposit and dissemination of scientific research documents, whether they are published or not. The documents may come from teaching and research institutions in France or abroad, or from public or private research centers.
L'archive ouverte pluridisciplinaire HAL, est destinée au dépôt et à la diffusion de documents scientifiques de niveau recherche, publiés ou non, émanant des établissements d'enseignement et de recherche français ou étrangers, des laboratoires publics ou privés.

\section{(ㄷ)(1) $\$$}

Distributed under a Creative Commons Attribution - NonCommerciall 4.0 International 


\section{CAS ANATOMO-CLINIQUE}

\section{Métastase mammaire d'un carcinome médullaire thyrö̈dien : un challenge}

\section{diagnostique}

\section{A misleading breast metastasis of medullary thyroid carcinoma}

Charlotte Gardair ${ }^{\mathrm{a}, \mathrm{b}}$, Philippe Drabent ${ }^{\mathrm{b}}$, Patrick Charveriat ${ }^{\mathrm{c}}$, Philippe Bertheau ${ }^{\mathrm{a}, \mathrm{b}}$, Laurence CahenDoidy $^{\mathrm{a}, \mathrm{d}}$, Anne de Roquancourt ${ }^{\mathrm{a}, \mathrm{b}}$

${ }^{a}$ Université Paris Diderot, Sorbonne-Paris-Cité, Paris, France

${ }^{\mathrm{b}}$ Service d'Anatomie et Cytologie Pathologiques, CHU Saint Louis, AP-HP, Paris, France

${ }^{\mathrm{c}}$ Centre des maladies du sein, CHU Saint Louis, AP-HP, Paris, France

${ }^{\mathrm{d}}$ Unité de chirurgie mammaire, CHU Saint Louis, AP-HP, Paris, France

* Auteur correspondant

Adresse e-mail : charlotte.gardair@aphp.fr

Téléphone : 0142499933

Fax : 0142494922

\section{Résumé}

La reconnaissance des métastases mammaires par le pathologiste est fondamentale car leur pronostic et leur traitement sont différents de ceux des carcinomes mammaires primitifs. Nous rapportons l'observation d'une patiente de 54 ans présentant sur sa mammographie de dépistage un nodule du sein gauche connu depuis 5 ans ayant discrètement augmenté de taille. L'échographie mammaire montrait une formation nodulaire hypoéchogène régulière de $1,2 \mathrm{~cm}$. Une microbiopsie a été effectuée. A l'examen microscopique, on observait une prolifération tumorale réalisant des massifs au sein d'un stroma peu abondant richement vascularisé. Les cellules tumorales avaient un cytoplasme moyennement abondant, éosinophile, granuleux et un noyau arrondi, légèrement atypique. Une mitose a été retrouvée en moyenne pour 10 champs au grossissement x400. Les cellules tumorales 
n'exprimaient pas les récepteurs hormonaux mais la chromogranine A, la synaptophysine, le TTF1 et la thyrocalcitonine. L'index de prolifération établi par l'anticorps anti-Ki67 était de 5\%. Le diagnostic retenu était celui de localisation secondaire d'une tumeur neuro-endocrine bien différenciée, dont le profil immunohistochimique oriente en premier lieu vers une origine thyroïdienne. Nous apprenions ultérieurement que la patiente avait un antécédent de thyroïdectomie totale 13 ans auparavant. Il s'agissait d'un carcinome médullaire de la thyroïde sporadique. Dans le cadre du bilan d'extension, la scintigraphie osseuse a mis en évidence une lésion lacunaire de la partie postérieure de l'aile iliaque droite suspecte de localisation secondaire. Cette lésion iliaque droite a été biopsiée. Il s'agissait également d'une localisation du carcinome médullaire thyroïdien connu. Le diagnostic final est celui de carcinome médullaire thyroïdien métastatique, lentement évolutif, la métastase mammaire existant probablement depuis 5 ans.

\section{Summary}

Recognition of mammary metastases by pathologists is fundamental because their prognosis and treatment are different from those of primary mammary carcinomas. We report the case of a 54-yearold woman presenting on her mammography a left breast nodule known for 5 years, having discreetly increased in size. Breast ultrasound showed a regular $1.2 \mathrm{~cm}$ hypoechogenic nodular formation. A microbiopsy was performed. On microscopic examination, we observed a tumor proliferation realizing nests within a small, richly vascularized stroma. The tumor cells had a moderately abundant, eosinophilic, granular cytoplasm and a rounded, slightly atypical nucleus. One mitosis was found for 10 fields at x400 magnification. Tumor cells did not express hormone receptors but chromogranin A, synaptophysin, TTF1 and thyrocalcitonin. The proliferation index established by the anti-Ki67 antibody was $5 \%$. The diagnosis was a secondary localization of a well-differentiated neuro-endocrine tumor which immunohistochemical profile firstly suggests a thyroid origin. We later learned that the patient had a history of total thyroidectomy 13 years ago. It was a sporadic medullary carcinoma of the thyroid. Bone scintigraphy revealed a lacunar lesion of the posterior part of the right iliac wing 
suspicious of secondary location. This right iliac lesion was biopsied. It was also a localization of the medullary thyroid carcinoma. The final diagnosis is a metastatic medullary thyroid carcinoma, slowly progressive, the mammary metastasis having probably existed for 5 years.

\section{Mots clés}

Sein ; Métastase ; Carcinome médullaire thyroïdien ; Neuroendocrine

\section{Keywords}

Breast ; Metastasis ; Medullary thyroid carcinoma ; Neuroendocrine

\section{Introduction}

Du fait de la fréquence élevée des cancers mammaires primitifs, de la rareté et de la non spécificité de l'aspect radiologique des métastases mammaires, une lésion mammaire de découverte récente est habituellement présumée être d'origine primitive. La reconnaissance des métastases mammaires par le pathologiste est très importante car leur pronostic et leur traitement sont différents de ceux des carcinomes mammaires primitifs.

\section{Observation}

Une femme de 54 ans présentait sur sa mammographie de dépistage un nodule du quadrant supéroexterne du sein gauche homogène, régulier, ayant discrètement augmenté de volume $(1,5 \mathrm{~cm}$ versus 1 cm 5 ans auparavant), sans prolongement spiculé ni microcalcification (Figure 1A). L'échographie mammaire montrait une formation nodulaire hypoéchogène du quadrant supéro-externe du sein gauche régulière de $1,2 \times 1 \times 0,8 \mathrm{~cm}$, sans atténuation acoustique postérieure (Figure 1B).

L'examen clinique était normal, sans masse palpable. Les aires ganglionnaires étaient libres. 
Une microbiopsie du sein gauche a été effectuée. L'examen microscopique montrait une prolifération tumorale réalisant des massifs au sein d'un stroma grêle riche en vaisseaux. Les cellules tumorales avaient un cytoplasme moyennement abondant, éosinophile, granuleux et un noyau arrondi, légèrement atypique (Figure 2A). Une mitose a été retrouvée en moyenne pour 10 champs au grossissement x 400. Il n'existait pas de lésion carcinomateuse in situ. La coloration au rouge congo ne mettait pas en évidence de stroma amyloïde.

L'étude immunohistochimique a permis les constatations suivantes :

- Anticorps anti-cytokératine AE1-AE3 : marquage cytoplasmique et membranaire intense et diffus des cellules tumorales

- Anticorps anti-récepteurs aux œstrogène et anti-récepteurs à la progestérone : absence de marquage des cellules tumorales

- Anticorps anti-chromogranine A : marquage cytoplasmique diffus des cellules tumorales (Figure 2B)

- Anticorps anti-synaptophysine : marquage cytoplasmique diffus des cellules tumorales

- Anticorps anti-GATA3: absence de marquage des cellules tumorales

- Anticorps anti-TTF1 : marquage nucléaire d'environ $70 \%$ des cellules tumorales

- Anticorps anti-thyrocalcitonine : marquage cytoplasmique diffus des cellules tumorales (Figure 2C)

- L'index de prolifération établi avec l'anticorps anti-ki67 était à $5 \%$

Le diagnostic retenu était celui de localisation secondaire mammaire gauche d'une tumeur neuroendocrine bien différenciée, dont le profil immunohistochimique oriente en premier lieu vers une origine thyroïdienne.

Nous apprenions ultérieurement que la patiente avait un antécédent de thyroïdectomie totale 13 ans auparavant. Il s'agissait d'un carcinome médullaire de la thyroïde sporadique du lobe thyroïdien droit, mesurant $3 \mathrm{~cm}$ de grand axe, n'atteignant pas la capsule thyroïdienne, pT2N0M0, traité par thyroïdectomie totale et curage ganglionnaire cervical bilatéral. Aucune métastase ganglionnaire n'avait été retrouvée sur un total de 24 ganglions lymphatiques examinés. Aucune mutation germinale de RET n'avait été mise en évidence. 
Dans le cadre du bilan d'extension, la scintigraphie osseuse a mis en évidence une lésion lacunaire isolée de $10 \mathrm{~mm}$ de la partie postérieure de l'aile iliaque droite suspecte de localisation secondaire. Cette lésion iliaque droite a été biopsiée. Il s'agissait également d'une localisation du carcinome médullaire thyroïdien connu.

Il a été décidé d'effectuer une tumorectomie mammaire gauche. L'analyse anatomopathologique de la pièce de tumorectomie a retrouvé une tumeur aux caractéristiques morphologiques et immunohistochimiques superposables à celle de la biopsie, confirmant le diagnostic de localisation secondaire du carcinome médullaire thyroïdien connu.

Au total, il s'agissait donc d'un carcinome médullaire thyroïdien métastatique, lentement évolutif, la métastase mammaire existant probablement depuis 5 ans. Une surveillance de la lésion osseuse de l'aile iliaque droite a été décidée.

\section{Discussion}

Les métastases mammaires sont rares et représentent environ $1 \%$ de l'ensemble des tumeurs malignes mammaires [1]. Ce sont des lésions importantes à diagnostiquer du fait d'un pronostic et d'un traitement très différents d'un cancer mammaire primitif. Les lymphomes, leucémies, mélanomes et les carcinomes sont à l'origine des principales métastases [2]. Parmi les carcinomes, il est décrit des métastases mammaires de cancers ovariens [2], pulmonaires [3], gastro-intestinaux, rénaux [4], ORL [5] et de tumeurs neuro-endocrines [6, 7]. Ces métastases mammaires sont communément situées dans le tissu sous-cutané superficiel, épargnent les structures ductulo-lobulaires et n'entraînent pas de rétraction mamelonnaire [1].

Les caractéristiques radiologiques des métastases mammaires ne sont pas spécifiques et correspondent le plus souvent à celles des tumeurs bénignes mammaires primitives. Elles sont en majorité bien limitées, rondes à ovalaires, très vascularisées, hypo-échogènes, sans microcalcification, sans spicule, 
et sans distorsion architecturale. La principale difficulté diagnostique vient du fait que les métastases mammaires se présentent dans $75 \%$ des cas comme une lésion unique [1,2], contrairement aux multiples lésions métastatiques observées dans d'autres organes tels que le foie et le poumon. Le diagnostic anatomopathologique des métastases mammaires est difficile, car elles peuvent présenter des caractéristiques morphologiques et immunohistochimiques similaires à celles des carcinomes mammaires primitifs. Cependant, l'absence de carcinome in situ, l'absence de calcification (sauf dans les adénocarcinomes ovariens séreux de haut grade avec possibles calcifications psammomateuses), la bonne limitation lésionnelle avec pseudo-capsule et refoulement du parenchyme mammaire adjacent, l'absence de métastase ganglionnaire axillaire, ainsi que la négativité des récepteurs hormonaux dans une tumeur bien différenciée doivent alerter sur la possibilité d'une métastase [1]. L'immunohistochimie peut aider dans certains cas en gardant en tête qu'aucun anticorps n'est 100\% sensible et spécifique : PS100, HMB45 et Melan A pour éliminer un mélanome (en sachant que la PS100 est exprimée dans environ 50\% des carcinomes mammaires primitifs), TTF1 pour l'origine pulmonaire ou thyroidienne, WT1 pour les adénocarcinomes ovariens séreux de haut grade, CDX2 pour l'origine gastro-intestinale, et enfin la mammaglobine, le GCDFP-15 et GATA3 en faveur de l'origine mammaire [1,8].

Aucune recommandation n'existe pour la prise en charge thérapeutique de ces métastases mammaires. Le pronostic des métastases mammaires est en général mauvais avec une survie médiane de 10 à 15 mois suivant le diagnostic [2,9]. Ce mauvais pronostic est lié au fait que la majorité des patients sont à un stade multi-métastatique avancé.

Les métastases mammaires de tumeurs neuroendocrines sont rares et représentent 1 à $2 \%$ de l'ensemble des métastases mammaires. Une centaine de cas ont été décrits dans la littérature. Il s'agit le plus souvent de tumeurs neuro-endocrines bien différenciées mais il peut également s'agir de carcinomes neuro-endocrines peu différenciés [6]. La rareté de ces tumeurs, un aspect morphologique proche de celui des carcinomes mammaires infiltrants (notamment du fait de l'architecture en nids et cordons), l'existence de carcinomes mammaires neuro-endocrines primitifs [10], et la possibilité d'une différenciation neuroendocrine dans les carcinomes mammaires primitifs peuvent être à l'origine 
d'erreurs diagnostiques. Or, poser un diagnostic de carcinome mammaire primitif au lieu de celui de métastase de tumeur neuro-endocrine peut entraîner des traitements agressifs injustifiés, surtout pour ces patients dont le pronostic est meilleur que pour celui des métastases d'autre origine. La distinction entre une métastase, un carcinome neuro-endocrine mammaire primitif, ou un carcinome mammaire primitif avec différenciation neuroendocrine est difficile, leurs caractéristiques morphologiques et immunohistochimiques étant proches. Les caractéristiques morphologiques devant faire évoquer une métastase de tumeur neuro-endocrine bien différenciée sont : une architecture organoïde en nids ou cordons, des noyaux uniformes peu atypiques aux membranes nucléaires régulières, et une chromatine d'aspect « poivre et sel ». En comparaison avec les métastases de tumeurs neuro-endocrines bien différenciées, les carcinomes neuro-endocrines mammaires primitifs et les carcinomes mammaires primitifs avec différenciation neuroendocrine présentent le plus souvent une composante carcinomateuse in situ, des atypies nucléaires plus importantes, des membranes nucléaires irrégulières et une activité mitotique plus élevée [6]. Dans le cadre de tumeurs neuroendocrines bien différenciées, l'immunohistochimie peut être utile pour distinguer une métastase d'une tumeur mammaire primitive : TTF-1 pour l'origine pulmonaire ou thyroïdienne (en sachant que TTF-1 est exprimé dans environ 80\% des métastases de tumeurs neuro-endocrines bien différenciées pulmonaires [11] et dans 90\% des métastases de carcinomes médullaires thyroïdiens [12]), CDX2 pour l'origine gastro-intestinale, PAX8 pour l'origine pancréatique ou gynécologique et enfin la mammaglobine, le GCDFP-15 et GATA3 pour l'origine mammaire [6,8]. Une expression de thyrocalcitonine évoque une origine thyroïdienne, mais n'est pas spécifique. En effet, il a été reporté une sécrétion de calcitonine dans des carcinomes neuroendocrines extra-thyroïdiens (d'origine pulmonaire, pancréatique, laryngé, vésical, mammaire ou ovarien) [13].

Dans la littérature, il a été décrit 20 cas de métastases mammaires de carcinome médullaire de la thyroïde. Ces métastases, unilatérales ou bilatérales, surviennent très majoritairement chez des femmes, avec un âge moyen de 42 ans au moment du diagnostic. L'intervalle entre la thyroïdectomie et la détection de la métastase mammaire est très variable allant de quelques mois à 28 ans, avec une moyenne à 6 ans $[2,14,15,16]$. 
Le carcinome médullaire de la thyroïde est un cancer rare (environ $3 \%$ des cancers de la thyroïde) qui se développe aux dépens des cellules $C$ thyroïdiennes responsables de la sécrétion de calcitonine. Un tiers des cancers médullaires de la thyroïde (CMT) sont des formes familiales : ils s'intègrent alors dans la néoplasie endocrinienne multiple de type 2, affection multiglandulaire héréditaire monogénique de transmission autosomique dominante rattachée à des mutations germinales du protooncogène RET, et dont la prévalence est estimée à 0,2/1000. La dissémination du carcinome médullaire de la thyroïde se fait par les voies lymphatique et sanguine (ganglions cervicaux, médiastinaux, poumon, foie, os). Le pronostic du carcinome médullaire de la thyroïde est essentiellement lié au stade anatomo-clinique et à la qualité de l'exérèse chirurgicale initiale. Les taux de survie globale à dix ans atteignent $80 \%$ pour les patients non biologiquement guéris par la chirurgie (c'est-à-dire présentant une élévation persistante de la calcitonine en post-opératoire) et $95 \%$ pour les patients biologiquement guéris en postopératoire [17].

\section{Conclusion}

Du fait de la fréquence élevée des cancers mammaires primitifs, de la rareté et de la non spécificité de l'aspect radiologique des métastases mammaires, une lésion mammaire de découverte récente est habituellement présumée être d'origine primitive. Cependant, la reconnaissance des métastases mammaires par le pathologiste est fondamentale afin d'éviter des traitements agressifs injustifiés, surtout dans le cadre du bon pronostic des tumeurs neuro-endocrines bien différenciées.

Une masse bien limitée, une morphologie inhabituelle, l'absence de composante carcinomateuse in situ, le profil immunohistochimique de type trigle négatif (d'autant plus sur une tumeur bien à moyennement différenciée) doivent alerter et faire évoquer une métastase mammaire.

\section{Légendes des figures}




\section{Figure 1}

A : mammographie de dépistage montrant un nodule dans le quadrant supérieur du sein gauche homogène, régulier de $1,5 \mathrm{~cm}$, sans prolongement spiculé ni microcalcification.

Mammography showing an homogeneous and regular nodule in the upper quadrant of the left breast, without spicular extension or microcalcification.

B : échographie mammaire montrant une formation nodulaire hypoéchogène régulière de $1,2 \times 1 \times 0,8$ $\mathrm{cm}$ du sein gauche, sans atténuation acoustique postérieure.

Mammary ultrasound showing a regular hypoechogenic nodular formation of $1.2 \times 1 \times 0.8$ centimeters of the left breast, without posterior acoustic attenuation.

\section{Figure 2}

Caractéristiques morphologiques et immunohistochimiques de la métastase mammaire du carcinome médullaire de la thyroïde :

Morphological and immunohistochemical characteristics of the breast metastasis of medullary carcinoma of the thyroid:

A : prolifération tumorale réalisant des massifs au sein d'un stroma grêle riche en vaisseaux. Les cellules tumorales avaient un cytoplasme moyennement abondant, éosinophile, granuleux et un noyau arrondi, légèrement atypique, coloration HES, grandissement X 200

A : tumor proliferation realizing nests within a small, richly vascularized stroma. The tumor cells had a moderately abundant, eosinophilic, granular cytoplasm and a rounded, slightly atypical nucleus

(HES, X200)

B : en immunohistochimie, grandissement X 300 : expression cytoplasmique diffuse de la chromogranine A par les cellules tumorales

B : cytoplasmic and diffuse expression of chromogranin A by tumor cells (immunochemistry, X300)

C : en immunohistochimie, grandissement X 300 : expression cytoplasmique diffuse de la thyrocalcitonine par les cellules tumorales

C : cytoplasmic and diffuse expression of thyrocalcitonin by tumor cells (immunochemistry, X300) 


\section{Bibliographie}

[1] Lee AH. The histological diagnosis of metastases to the breast from extramammary malignancies. J Clin Pathol 2007;60:1333-1341.

[2] DeLair DF, Corben AD, Catalano JP, Vallejo CE, Brogi E, Tan LK. Non-mammary metastases to the breast and axilla: a study of 85 cases. Mod Pathol. $2013 ; 26: 343-9$.

[3] Ali RH, Taraboanta C, Mohammad T, Hayes MM, Ionescu DN. Metastatic non-small cell lung carcinoma a mimic of primary breast carcinoma - case series and literature review. Virchows Arch 2017.

[4] Xu Y, Hou R, Lu Q, Deng Y, Hu B. Renal clear cell carcinoma metastasis to the breast ten years after nephrectomy: a case report and literature review. Diag Pathol 2017;12:76.

[5] Longo R, Melgar E, Campitiello M, Plastino F, Eid N, Quirin I, et al. Breast metastasis from squamous cell carcinoma of the oropharynx: a case report. J Med Case Rep 2017;11:355.

[6] Mohanty SK, Kim SA, DeLair DF, Bose S, Laury AR, Chopra S et al. Comparison of metastatic neuroendocrine neoplasms to the breast and primary invasive mammary carcinomas with neuroendocrine differentiation. Modern Pathology 2016;29:788-798.

[7] Battistella M, Hamy AS, Janin A, de Roquancourt A. Breast metastasis of ileal endocrine carcinoma. Ann Pathol 2011;31:112-114.

[8] Perry KD, Reynolds C, Rosen DG, Edgerton ME, Albarracin CT, Gilcrease MZ. Metastatic neuroendocrine tumour in the breast: a potential mimic of in-situ and invasive mammary carcinoma. Histopathology 2011;59:619-630

[9] Williams SA, Ehlers RA, Hunt KK, Yi M, Kuerer HM, Singletary SE et al. Metastases to the breast from non breast solid neoplasms: presentation and determinants of survival. Cancer 2007;110:731-737.

[10] Visscher DW, Yasir S. Neuroendocrine Tumors of the Breast. Endocr Pathol. 2017;28(2):121127. 
[11] Oliveira AM, Tazelaar HD, Myers JL, Erickson LA, Lloyd RV. Thyroid transcription factor-1 distinguishes metastatic pulmonary from well differentiated NE tumors of others sites. Am J Surg Pathol 2001;25:815-819.

[12] Ordonez NG. Thyroid transcription factor-1 is a marker of lung and thyroid carcinomas. Adv Anat Pathol. 2000;7:123-127.

[13] Vahidi S, Stewart J, Amin K, Racila E, Li F. Metastatic medullary thyroid carcinoma or calcitonin secreting carcinoid tumor of lung? A diagnostic dilemma in a patient with lung mass and thyroid nodule. Diagnostic Cytopathology. 2017;46(4):345-348.

[14] Mandanas S, Margaritidou E, Christoforidou V, Karoglou E, Geranou C, Chrisoulidou A et al. Breast metastasis from medullary thyroid carcinoma in a male patient: case report and review of the literature. Rare Tumors 2015;7:5765.

[15] Martins Filho SN , Giannotti MA, Siqueira SAC. Medullary carcinoma of the thyroid metastatic to the breast: a case report and literature review. Autopsy and Case Reports 2014; 4: 53-57.

[16] Hassan A, Siddiqui M, Jahangir S. A rare case of medullary carcinoma thyroid metastasizing to bilateral breast parenchyma. Nuclear Medicine Review 2016, Suppl B: B17-B19.

[17] Wells SA, Asa SL, Dralle H, Elisei R, Evans DB, Gagel RF et al. Revised American Thyroid Association guidelines for the management of medullary thyroid carcinoma. Thyroid. 2015;25:567610. 


\section{A}

B

(4)

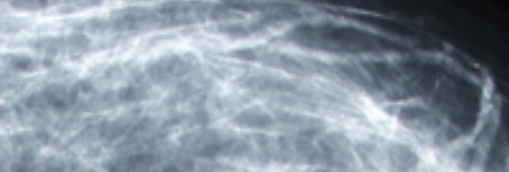

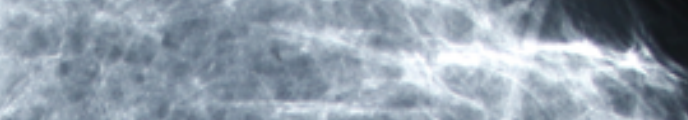

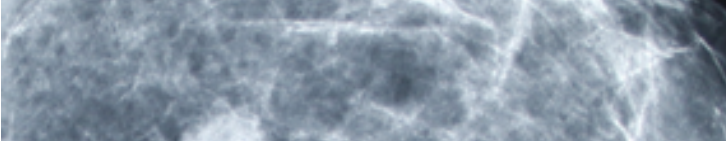

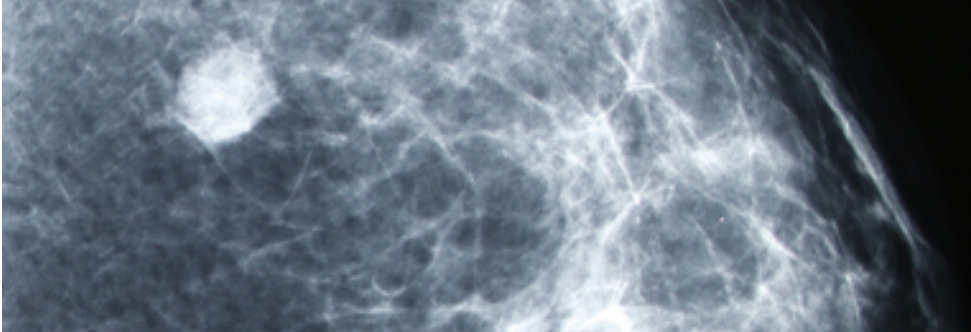

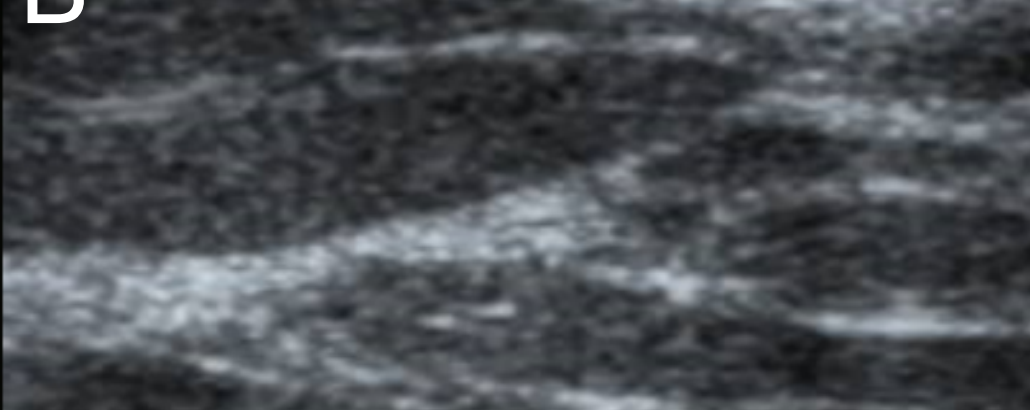

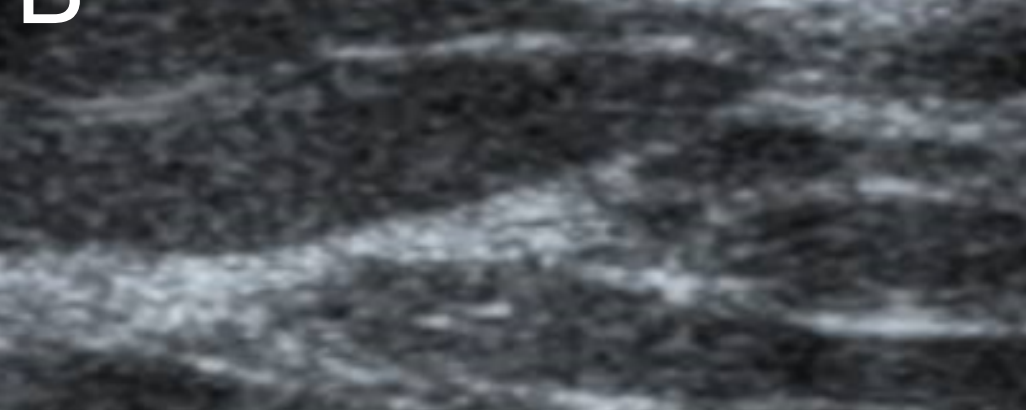

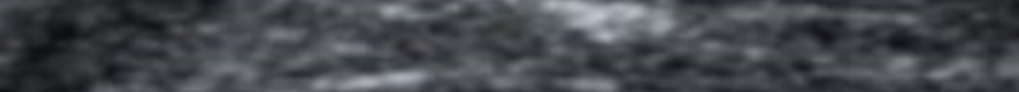

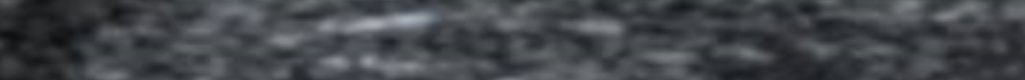

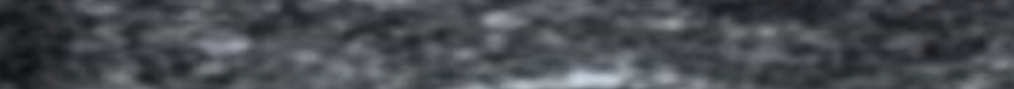

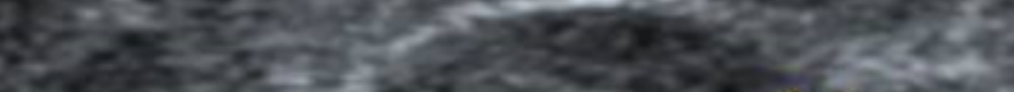

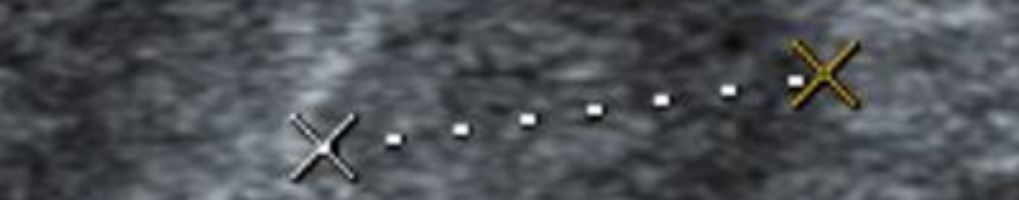

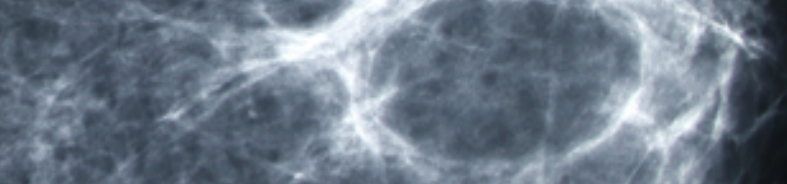

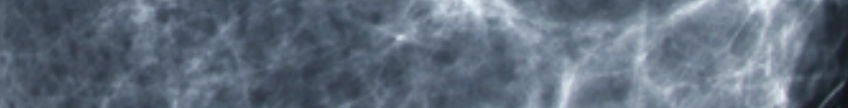

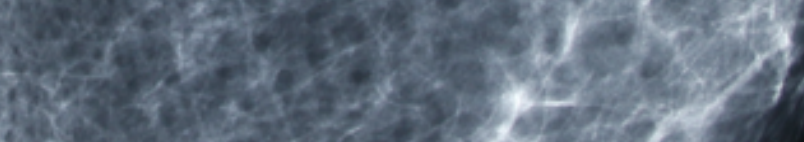

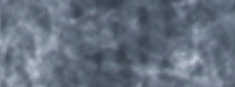

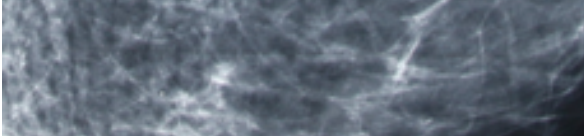

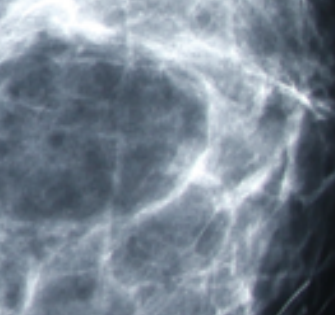

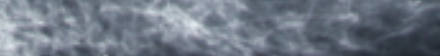

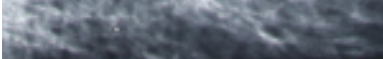

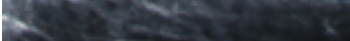

\section{Cos}
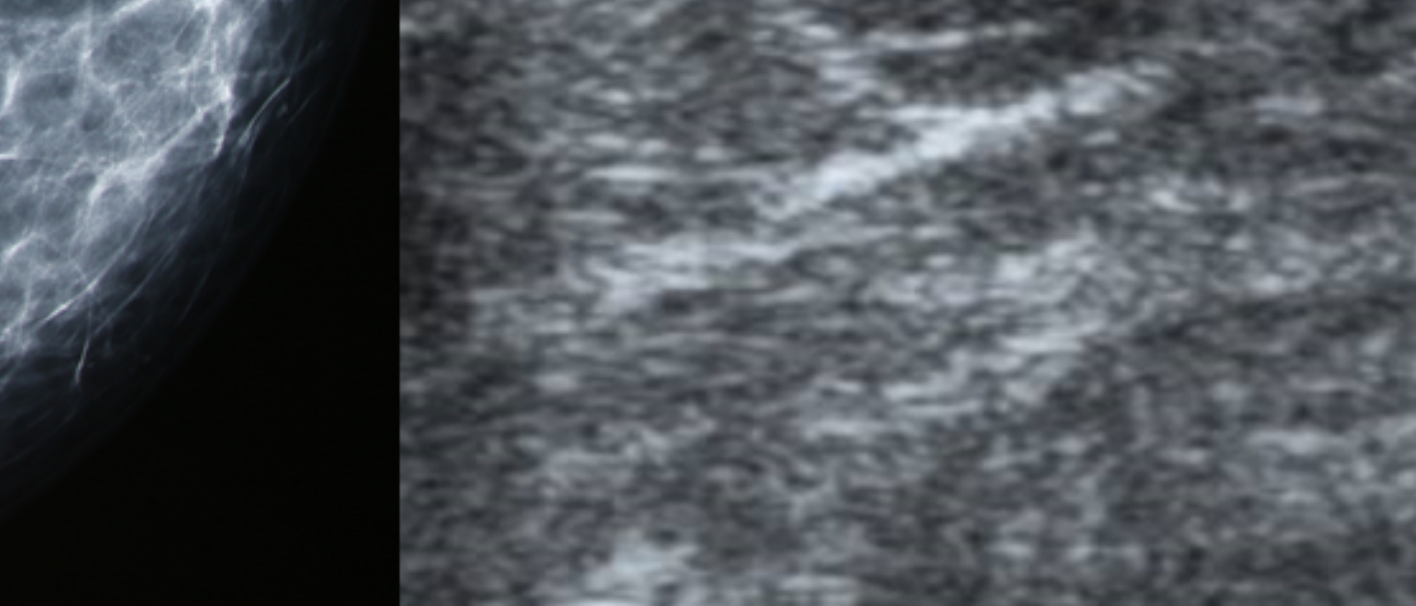


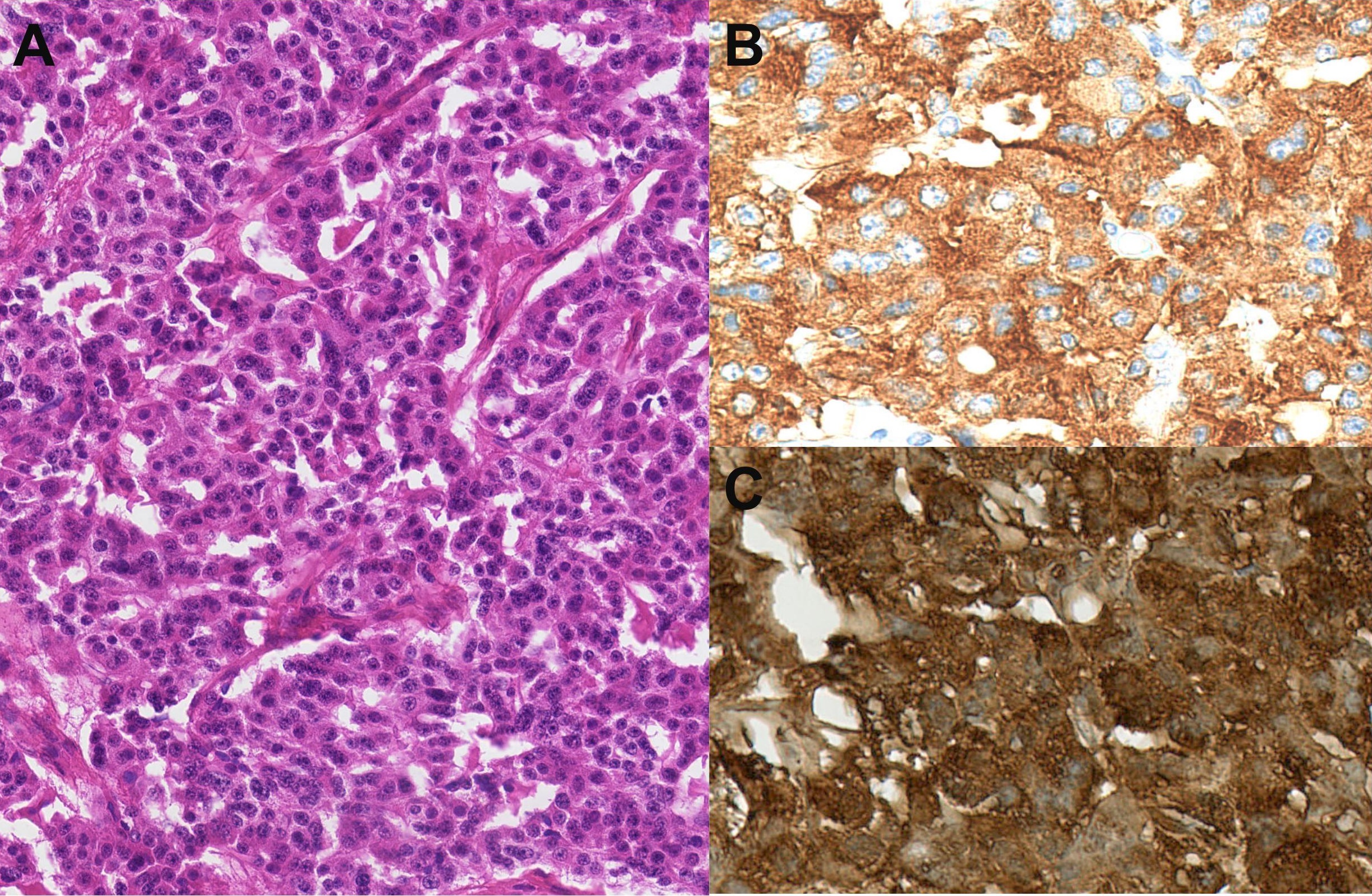

